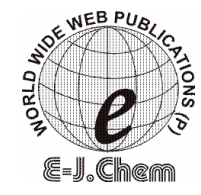

http://www.e-journals.net
ISSN: 0973-4945; CODEN ECJHAO

E-Journal of Chemistry

Vol. 5, No.1, pp. 144-148, January 2008

\title{
Synthesis and Antimicrobial Activity of Some New Chalcones of 2-Acetyl Pyridine
}

\author{
Y. RAJENDRA PRASAD*, P. PRAVEEN KUMAR, \\ P. RAVI KUMAR and A. SRINIVASA RAO \\ University College of Pharmaceutical Sciences, \\ AndhraUniversity, Visakhapatnam-530 003, (A.P), India. \\ dryrp@ rediffmail.com
}

Received 17 July 2007; Accepted 20 September 2007

\begin{abstract}
Six new chalcones were synthesised by condensing 2-acetyl pyridine with aldehyde derivatives in dilute ethanolic potassium hydroxide solution at room temperature according to Claisen-Schmidt condensation. All these compounds were characterised by means of their IR, ${ }^{1} \mathrm{H}$ NMR spectroscopic data and microanalyses. The antimicrobial activity of these compounds was evaluated by the cup plate method.
\end{abstract}

Keywords: Chalcones, Synthesis, Antimicrobial activity.

\section{Introduction}

Chalcones either natural or synthetic are known to exhibit various biological activities. They have been reported to possess antioxidant, ${ }^{1-4}$ antimalarial, ${ }^{5}$ antileishmanial, ${ }^{6}$ antiinflammatory, ${ }^{7}$ antitumor ${ }^{8}$ and antibacterial activity ${ }^{9}$. The presence of a reactive $\alpha, \beta$-unsaturated keto function in chalcones is found to be responsible for their antimicrobial activity, which may be altered depending on the type and position of substituent on the aromatic rings. In the present communication we report the reaction of 2-acetyl pyridine with different aromatic aldehydes to form chalcones (1-6). The structures of the various synthesized compounds were assigned on the basis of elemental analyses, IR and ${ }^{1} \mathrm{H}$ NMR spectral data. These compounds were also screened for their antimicrobial activity.

\section{Experimental}

Melting points were determined on a capillary melting point apparatus and are uncorrected. ${ }^{1} \mathrm{H}$ NMR spectra was recorded in the indicated solvent on Bruker WM $400 \mathrm{MHz}$ spectrometer with TMS as internal standard. Infrared spectra were recorded in $\mathrm{KBr}$ on Perkin-Elmer AC-1 spectrophotometer. Microanalyses were performed on Carlo Erba EA- 
1108 element analyzer and were within the $\pm 0.5 \%$ of the theoretical values. Column chromatography was performed on silica gel (Merck, 60-120 mesh).

\section{General procedure for the preparation of chalcones (1-6)}

A mixture of 2 -acetyl pyridine $(0.01 \mathrm{~mol})$ and aryl aldehyde $(0.01 \mathrm{~mol})$ was stirred in ethanol $(30 \mathrm{~mL})$ and then an aqueous solution of $\mathrm{KOH}(40 \%, 15 \mathrm{~mL})$ added to it. The mixture was kept overnight at room temperature and then it was poured into crushed ice and acidified with $\mathrm{HCl}$. The solid separated was filtered and crystallized from ethanol (Scheme 1). The Characterisation data of these compounds is described in Table-1 and 2.

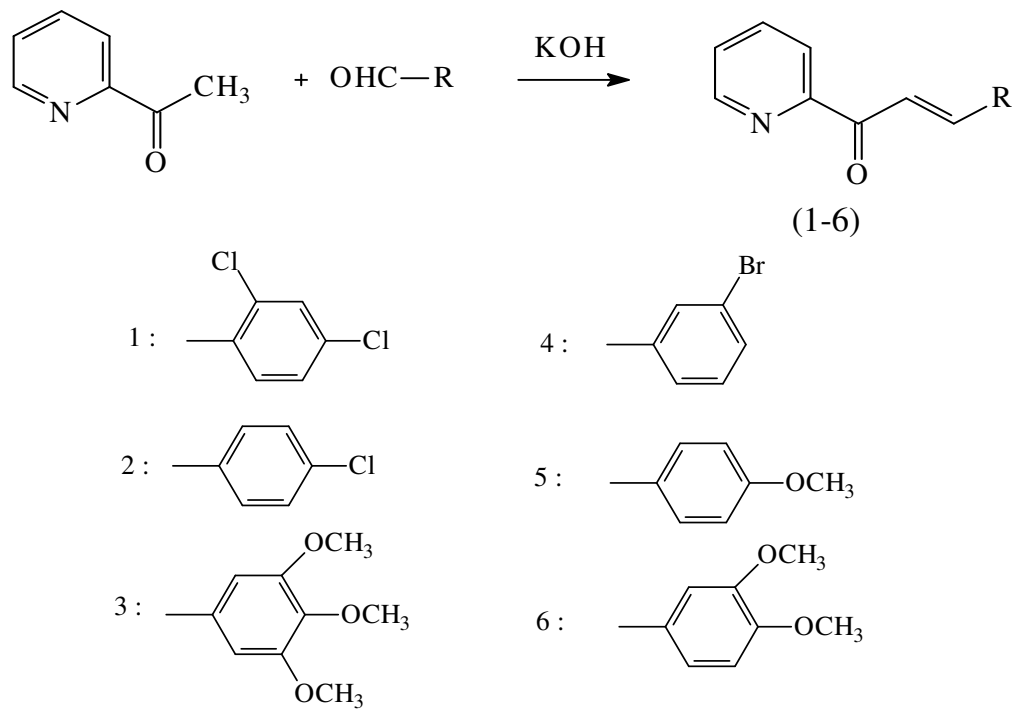

Scheme 1. Synthesis of chalcones of 2-acetyl pyridine

Table 1. Physical data of compounds (1-6)

\begin{tabular}{|c|c|c|c|c|c|c|c|c|c|}
\hline \multirow{3}{*}{ Compd. } & \multirow{3}{*}{ M.F. } & \multirow{3}{*}{$\begin{array}{l}\text { M.P. } \\
{ }^{\circ} \mathrm{C}\end{array}$} & \multirow{3}{*}{$\begin{array}{c}\text { Yield, } \\
\%\end{array}$} & \multicolumn{6}{|c|}{ Elemental analyses, $\%$} \\
\hline & & & & \multicolumn{2}{|c|}{$\mathrm{C}$} & \multicolumn{2}{|c|}{$\mathrm{H}$} & \multicolumn{2}{|c|}{$\mathrm{O}$} \\
\hline & & & & Found & Calcd & Found & Calcd. & Found & Calcd. \\
\hline 1 & $\mathrm{C}_{14} \mathrm{H}_{9} \mathrm{ONCl}_{2}$ & 155 & 88 & 60.23 & 60.45 & 3.12 & 3.26 & 5.36 & 5.75 \\
\hline 2 & $\mathrm{C}_{14} \mathrm{H}_{10} \mathrm{ONCl}$ & 167 & 82 & 69.25 & 69.00 & 4.02 & 4.13 & 6.32 & 6.56 \\
\hline 3 & $\mathrm{C}_{17} \mathrm{H}_{17} \mathrm{O}_{4} \mathrm{~N}$ & 115 & 78 & 68.04 & 68.24 & 5.32 & 5.68 & 21.10 & 21.38 \\
\hline 4 & $\mathrm{C}_{14} \mathrm{H}_{10} \mathrm{OBr}$ & 140 & 92 & 58.61 & 58.37 & 3.41 & 3.47 & 5.18 & 5.55 \\
\hline 5 & $\mathrm{C}_{15} \mathrm{H}_{13} \mathrm{O}_{2} \mathrm{~N}$ & 126 & 80 & 75.12 & 75.32 & 5.12 & 5.43 & 13.48 & 13.37 \\
\hline 6 & $\mathrm{C}_{16} \mathrm{H}_{15} \mathrm{O}_{3} \mathrm{~N}$ & 138 & 75 & 71.61 & 71.39 & 5.32 & 5.57 & 17.51 & 17.83 \\
\hline
\end{tabular}

\section{Antimicrobial activity}

Cup plate method ${ }^{10,11}$ using Mueller-Hinton agar medium was employed to study the preliminary antibacterial activity of (1-6) against B. pumilis, B. substilis, E.coli. and $P$. Vulgaris. The agar medium was purchased from HI media Laboratories Ltd., Mumbai, India. Preparation of nutrient broth, subculture, base layer medium, agar medium and peptone water was done as per the standard procedure. Each test compound $(5 \mathrm{mg})$ was dissolved in $5 \mathrm{~mL}$ of dimethyl sulfoxide $(1000 \mu \mathrm{g} / \mathrm{mL})$. Volumes of $0.05 \mathrm{~mL}$ and $0.1 \mathrm{~mL}$ of each compound were used for testing. 
Table 2. Spectral data of the compounds (1-6)

\begin{tabular}{|c|c|c|}
\hline Compd. & IR $\left(\mathrm{KBr}, \mathrm{cm}^{-1}\right)$ & ${ }^{1} \mathrm{H} \mathrm{NMR}\left(\mathrm{CDCl}_{3}, \mathrm{ppm}\right)$ \\
\hline 1 & $\begin{array}{l}1721(\mathrm{C}=\mathrm{O}) \\
1645(\mathrm{CH}=\mathrm{CH}) \\
855(\mathrm{C}-\mathrm{Cl}) \\
1512(\mathrm{C}=\mathrm{N})\end{array}$ & $\begin{array}{l}7.31(1 \mathrm{H}, \mathrm{d}, \mathrm{C}-3-\mathrm{H}), 7.47(1 \mathrm{H}, \mathrm{d},-\mathrm{CO}-\mathrm{CH}=), 7.57 \\
(1 \mathrm{H}, \mathrm{d}, \mathrm{C}-6-\mathrm{H}), 7.88(1 \mathrm{H}, \mathrm{s}, \mathrm{C}-3-\mathrm{H}), 7.92(2 \mathrm{H}, \mathrm{m}, \\
\left.\mathrm{C}^{\prime}-4^{\prime}-\mathrm{H}, \mathrm{C}-5^{\prime}-\mathrm{H}\right), 8.23(1 \mathrm{H}, \mathrm{d}, \mathrm{C}-5-\mathrm{H}), 8.32(1 \mathrm{H}, \mathrm{d}, \\
=\mathrm{CH}-\mathrm{Ar}), 8.78(1 \mathrm{H}, \mathrm{d}, \mathrm{C}-6-\mathrm{H})\end{array}$ \\
\hline 2 & $\begin{array}{l}1724(\mathrm{C}=\mathrm{O}), \\
1648(-\mathrm{CH}=\mathrm{CH}), \\
851(\mathrm{C}-\mathrm{Cl}) \\
1536(\mathrm{C}=\mathrm{N})\end{array}$ & $\begin{array}{l}7.40(1 \mathrm{H}, \mathrm{d},-\mathrm{CO}-\mathrm{CH}=), 7.55\left(1 \mathrm{H}, \mathrm{m}, \mathrm{C}-4^{\prime}-\mathrm{H}\right), 7.69 \\
(1 \mathrm{H}, \mathrm{d},=\mathrm{CH}-\mathrm{Ar}), 7.89\left(1 \mathrm{H}, \mathrm{d}, \mathrm{C}-3^{\prime}-\mathrm{H}\right), 7.96(1 \mathrm{H}, \mathrm{m}, \\
\left.\mathrm{C}-5^{\prime}-\mathrm{H}\right), 8.22(2 \mathrm{H}, \mathrm{d}, \mathrm{C}-2-\mathrm{H}, \mathrm{C}-6-\mathrm{H}), 8.34(2 \mathrm{H}, \mathrm{d}, \mathrm{C}- \\
3-\mathrm{H}, \mathrm{C}-5-\mathrm{H}), 8.78\left(1 \mathrm{H}, \mathrm{d}, \mathrm{C}-6^{\prime}-\mathrm{H}\right)\end{array}$ \\
\hline 3 & $\begin{array}{l}1725(\mathrm{C}=\mathrm{O}), \\
1650(-\mathrm{CH}=\mathrm{CH}), \\
1172(-\mathrm{OCH})_{3}, \\
1548(\mathrm{C}=\mathrm{N})\end{array}$ & $\begin{array}{l}3.90-3.94\left(9 \mathrm{H}, \mathrm{s}, 3 \mathrm{x}-\mathrm{OCH}_{3}\right), 7.30(2 \mathrm{H}, \mathrm{d}, \mathrm{C}-3-\mathrm{H}, \mathrm{C}- \\
\left.5^{\prime}-\mathrm{H}\right), 7.45(1 \mathrm{H}, \mathrm{d},-\mathrm{CO}-\mathrm{CH}=), 7.86(2 \mathrm{H}, \mathrm{s}, \mathrm{C}-2-\mathrm{H}, \\
\mathrm{C}-6-\mathrm{H}), 7.90\left(1 \mathrm{H}, \mathrm{m}, \mathrm{C}-4^{\prime}-\mathrm{H}\right), 8.26(1 \mathrm{H}, \mathrm{d},=\mathrm{CH}- \\
\text { Ar }), 8.76\left(1 \mathrm{H}, \mathrm{d}, \mathrm{C}-6^{\prime}-\mathrm{H}\right)\end{array}$ \\
\hline 4 & $\begin{array}{l}1730(\mathrm{C}=\mathrm{O}), \\
1652(-\mathrm{CH}=\mathrm{CH}), \\
860(\mathrm{C}-\mathrm{Br}) \\
1530(\mathrm{C}=\mathrm{N})\end{array}$ & $\begin{array}{l}7.21(1 \mathrm{H}, \mathrm{d},-\mathrm{CO}-\mathrm{CH}=), 7.29\left(1 \mathrm{H}, \mathrm{d}, \mathrm{C}-3^{\prime}-\mathrm{H}\right), 7.39 \\
(1 \mathrm{H}, \mathrm{s}, \mathrm{C}-2-\mathrm{H}), 7.60\left(1 \mathrm{H}, \mathrm{d}, \mathrm{C}-4^{\prime}-\mathrm{H}\right), 7.68(1 \mathrm{H}, \mathrm{m}, \\
\left.\mathrm{C}-5^{\prime}-\mathrm{H}\right), 7.85(1 \mathrm{H}, \mathrm{d}, \mathrm{C}-6-\mathrm{H}), 8.18(1 \mathrm{H}, \mathrm{m}, \mathrm{C}-5-\mathrm{H}) \text {, } \\
8.29(1 \mathrm{H}, \mathrm{d},=\mathrm{CH}-\mathrm{Ar}), 8.62(1 \mathrm{H}, \mathrm{d}, \mathrm{C}-4-\mathrm{H}), 8.77 \\
\left(1 \mathrm{H}, \mathrm{d}, \mathrm{C}-6^{\prime}-\mathrm{H}\right)\end{array}$ \\
\hline 5 & $\begin{array}{l}1732(\mathrm{C}=\mathrm{O}), \\
1651(-\mathrm{CH}=\mathrm{CH}), \\
1174(-\mathrm{OCH})_{3}, \\
1584(\mathrm{C}=\mathrm{N})\end{array}$ & $\begin{array}{l}3.89\left(3 \mathrm{H}, \mathrm{s},-\mathrm{OCH}_{3}\right), 6.99(1 \mathrm{H}, \mathrm{d},-\mathrm{CO}-\mathrm{CH}=), \\
7.94(1 \mathrm{H}, \mathrm{d},=\mathrm{CH}-\mathrm{Ar}), 8.02\left(1 \mathrm{H}, \mathrm{m}, \mathrm{C}-4^{\prime}-\mathrm{H}\right), 8.10 \\
(2 \mathrm{H}, \mathrm{d}, \mathrm{C}-2-\mathrm{H}, \mathrm{C}-6-\mathrm{H}), 8.48\left(1 \mathrm{H}, \mathrm{m}, \mathrm{C}-5^{\prime}-\mathrm{H}\right), 8.52 \\
\left(1 \mathrm{H}, \mathrm{m}, \mathrm{C}-3^{\prime}-\mathrm{H}\right), 8.65(2 \mathrm{H}, \mathrm{d}, \mathrm{C}-3-\mathrm{H}, \mathrm{C}-5-\mathrm{H}), 9.10 \\
\left(1 \mathrm{H}, \mathrm{d}, \mathrm{C}-6^{\prime}-\mathrm{H}\right)\end{array}$ \\
\hline 6 & $\begin{array}{l}1735(\mathrm{C}=\mathrm{O}), \\
1649(-\mathrm{CH}=\mathrm{CH}), \\
1170(-\mathrm{OCH})_{3}, \\
1412(\mathrm{C}=\mathrm{N})\end{array}$ & $\begin{array}{l}3.89-3.93\left(6 \mathrm{H}, \mathrm{s}, 2 \mathrm{x}-\mathrm{OCH}_{3}\right), 7.10(1 \mathrm{H}, \mathrm{d},-\mathrm{CO}- \\
\mathrm{CH}=), 7.30\left(1 \mathrm{H}, \mathrm{d}, \mathrm{C}-3^{\prime}-\mathrm{H}\right), 7.62\left(1 \mathrm{H}, \mathrm{m}, \mathrm{C}-4^{\prime}-\mathrm{H}\right), \\
7.84(1 \mathrm{H}, \mathrm{d},=\mathrm{CH}-\mathrm{Ar}), 7.98\left(1 \mathrm{H}, \mathrm{m}, \mathrm{C}-5^{\prime}-\mathrm{H}\right), 8.12 \\
(1 \mathrm{H}, \mathrm{s}, \mathrm{C}-2-\mathrm{H}), 8.16(2 \mathrm{H}, \mathrm{d}, \mathrm{C}-5-\mathrm{H}, \mathrm{C}-6-\mathrm{H}), 8.73 \\
\left(1 \mathrm{H}, \mathrm{d}, \mathrm{C}-6^{\prime}-\mathrm{H}\right),\end{array}$ \\
\hline
\end{tabular}

Same cup plate method using PDA medium was employed to study the preliminary antifungal activity of (1-6) against $A$. niger and $P$. crysogenium. The PDA medium was purchased from HI media Laboratories Ltd., Mumbai, India. Preparation of nutrient broth, subculture, base layer medium and PDA medium was done as per the standard procedure. Each test compound $(5 \mathrm{mg})$ was dissolved in $5 \mathrm{~mL}$ of dimethyl sulfoxide $(1000 \mu \mathrm{g} / \mathrm{mL})$. Volumes of $0.05 \mathrm{~mL}$, and $0.1 \mathrm{~mL}$ of each compound were used for testing.

The cups each of $9 \mathrm{~mm}$ diameter were made by scooping out medium with a sterilized cork borer in a Petri dish which was streaked with the organisms. The solutions of each test compound $(0.05$ and $0.1 \mathrm{~mL})$ were added separately in the cups and Petri dishes were subsequently incubated. Benzyl Penicillin and Fluconazole were used as standard reference drugs ( $200 \& 500 \mu \mathrm{g} / \mathrm{mL}$ respectively) and Dimethyl Sulphoxide as a control which did not reveal any inhibition. Zone of inhibition produced by each compound was measured in $\mathrm{mm}$ and the results are presented in Table 3 and 4. 
Table 4. Antifungal activity of chalcones (1-6)

\begin{tabular}{cccccccccccccccc}
\hline & \multicolumn{1}{c}{1} & \multicolumn{3}{c}{2} & \multicolumn{3}{c}{3} & \multicolumn{3}{c}{5} & \multicolumn{3}{c}{6} & $\mathrm{C}$ & $\mathrm{S}$ \\
Organisms & 0.05 & 0.1 & 0.05 & 0.1 & 0.05 & 0.1 & 0.05 & 0.1 & 0.05 & 0.1 & 0.05 & 0.1 & & 0.05 \\
& $\mathrm{~mL}$ & $\mathrm{~mL}$ & $\mathrm{~mL}$ & $\mathrm{~mL}$ & $\mathrm{~mL}$ & $\mathrm{~mL}$ & $\mathrm{~mL}$ & $\mathrm{~mL}$ & $\mathrm{~mL}$ & $\mathrm{~mL}$ & $\mathrm{~mL}$ & $\mathrm{~mL}$ & & $\mathrm{~mL}$ \\
A. niger & 13 & 15 & 09 & 13 & -- & 10 & 08 & 12 & -- & 11 & 07 & 11 & -- & 18 \\
Pencillium & 12 & 16 & 08 & 14 & -- & 10 & 06 & 13 & -- & 12 & 06 & 11 & -- & 20 \\
crysogenium & & & & & & & & & & & & & & \\
\hline
\end{tabular}

C: Control (DMSO); S: Standard (Fluconozole) 


\section{Conclusion}

The screening results revealed that the compounds 1-6 showed significant antimicrobial activity. In particular compounds 3, 6, 5, 2 showed moderate to considerable antibacterial activity against all the organisms employed at a conc. of $1000 \mu \mathrm{g} / \mathrm{mL}(0.1 \mathrm{~mL}$ dose level $)$ and are comparable to that of standard drug Benzyl Penicillin. Similarly compounds 1, 2, 4, 5 showed moderate to considerable antifungal activity against all the organisms employed at a conc. of $1000 \mu \mathrm{g} / \mathrm{mL}(0.1 \mathrm{~mL}$ dose level) and are comparable to that of standard drug Fluconazole.

\section{Acknowledgements}

We are thankful to the Head, Sophisticated Instrumentation Facility, IISC, Bangalore for ${ }^{1} \mathrm{H}$ NMR Spectra and to Sipra Laboratories, Hyderabad for IR Spectra.

\section{References}

1. John Anto R, Sukumaran K, Kuttan G, Rao M N A, Subbaraju V and Kuttan R,. Cancer Letters. 1995, 97, 33.

2. Vaya R, Belinky P A and Aviram M, Free Radic. Biol. Med. 1997, 23, 302.

3. Mukherjee S, Kumar V, Prasad A K, Raj H G, Brakhe M E, Olsen C E, Jain S C and Parmar V P, Bioorg. Med. Chem. 2001, 9, 337.

4. Indyah S A, Timmerman H, Samhoedi M, Sastrohami D, Sugiyanto H and Van Der Goot H, Eur. J. Med. Chem. 2000, 35, 449.

5. Chen M, Christensen S B, Zhai L, Rasmussen M H, Theander T G, Frokjaer S, Steffensen B, Davidson J and Kharazmi A, J. Infect. Dis. 1997, 176, 1327.

6. Nielsen S F, Christensen S B, Cruciani G, Kharazmi A and Liljefors T, J. Med. Chem. 1998, 41, 4819.

7. Hsin-kaw H, Tai-Hua L, Pyang Wang J, Jey-Jeng W and Chun-Nan L, Pharm. Res. 1998, 15, 39.

8. Kumar S K, Hager E, Catherine P, Gurulingappa H, Davidson N E and Khan S R, J. Med. Chem. 2003, 46, 2813.

9. Prasad Y R, Prasoona L, Rao A L, Lakshmi K, Kumar P R and Rao B, G. Int. J. Chem. Sci. 2005, 3(4), 685-689.

10. Banty A L, The Antimicrobial Susceptibility test; Principle and practice, edited by Illus lea and Febiger, (Philadelphia, Pa USA), 1976, 180 .

11. Seely H W and Van Demark P J, Microbes in action: A laboratory manual of Microbiology, D.B. Taraporewala Sons and Co, Bombay, 1975, 55. 


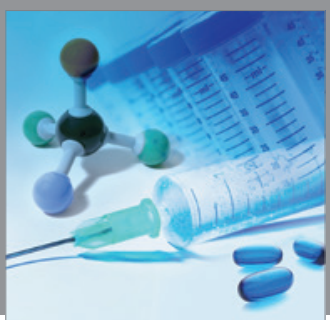

International Journal of

Medicinal Chemistry

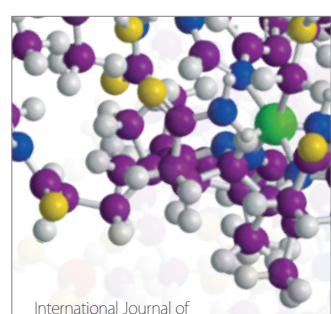

Carbohydrate Chemistry

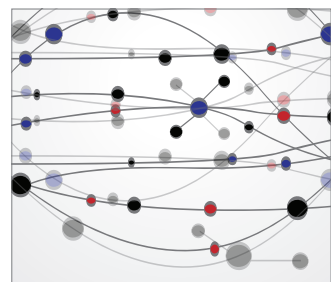

The Scientific World Journal
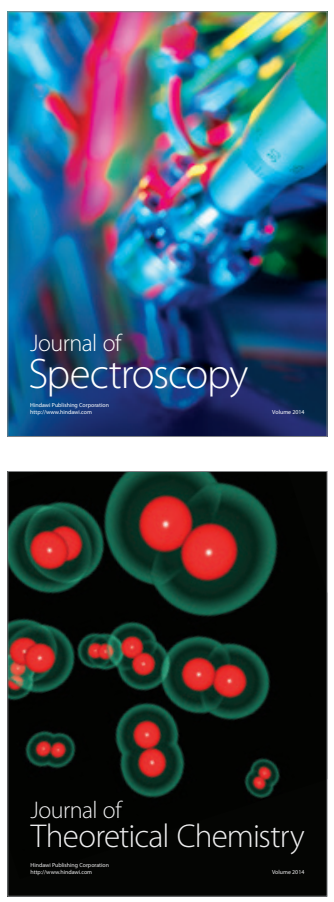
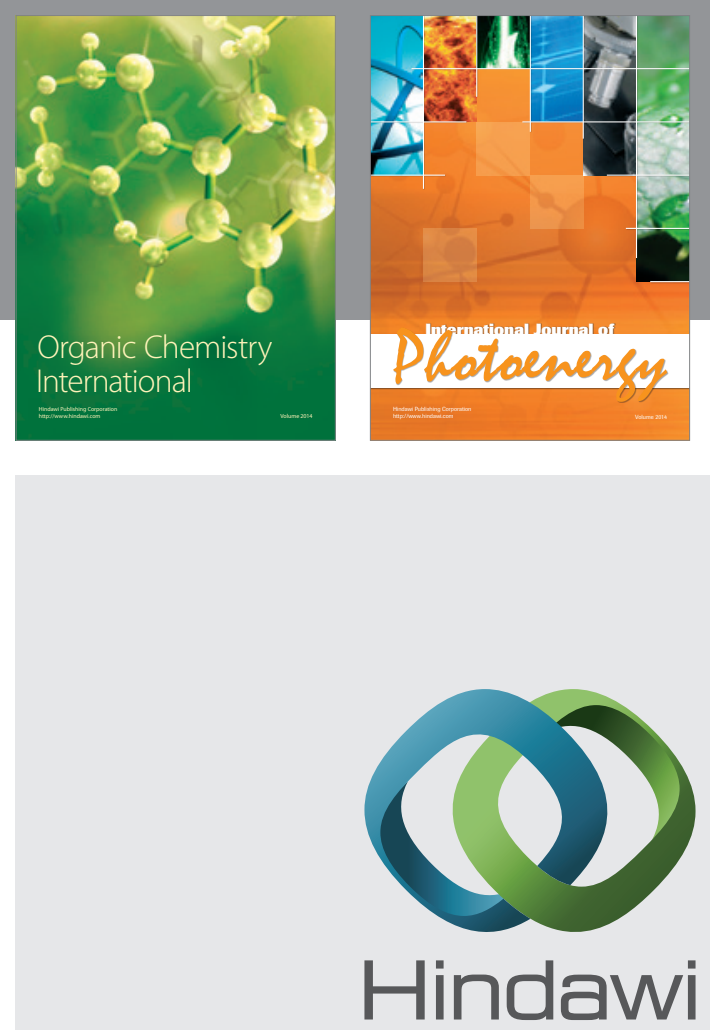

Submit your manuscripts at

http://www.hindawi.com
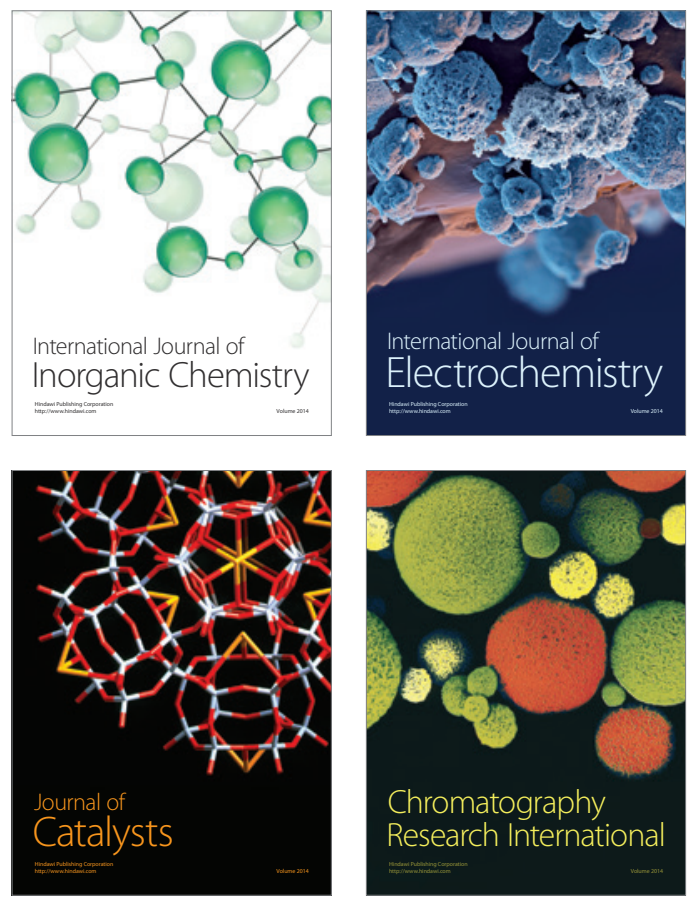
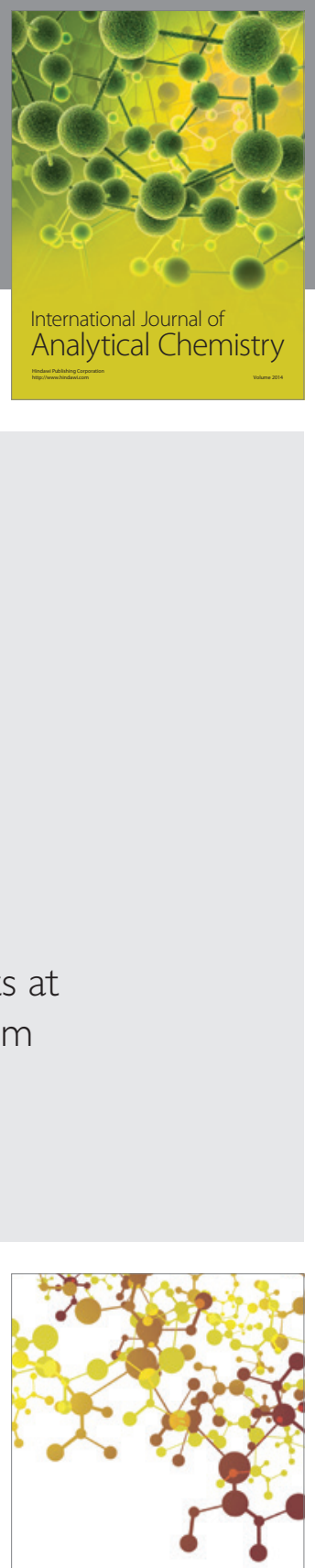

Journal of

Applied Chemistry
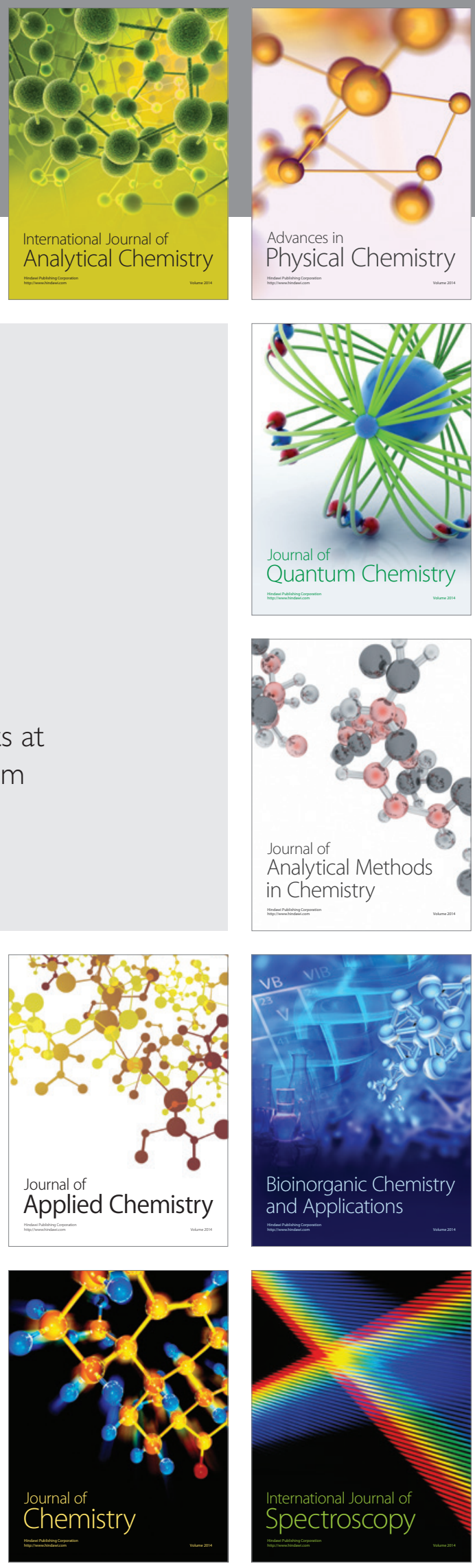